\title{
Contribution à l'étude des races d'oïdium du haricot (Erysiphe polygoni D.C.) en Guadeloupe
}

\author{
P. Pauvert
}

INRA, Station de Pathologie végétale, de Phytoécologie et de Malherbologie, CRAAG, Domaine Duclos, BP 1232, 97184 Pointe-àPitre Cedex, France

(reçu le 9-8-1988, accepté le 12-12-1988)

Résumé - L'hypothèse d'une hétérogénéité de la population d'oïdium (Erysiphe polygoni D.C.) en Guadeloupe, suggérée par l'observation de la maladie en serre et au champ sur diverses variétés de haricot, a été vérifiée par inoculation en pièces climatiques de variétés différentielles. Deux races physiologiques d'oïdium ont pu être mises en évidence.

En culture, la race 1 apparaît au début de la saison la plus favorable à la maladie (décembre-mai), son développement est rapide tant que les températures maximales ne dépassent pas $30^{\circ} \mathrm{C}$. La race 2 apparaît plus tardivement, affecte avec une plus ou moins grande aggressivité un plus grand nombre de variétés et supporte mieux les températures élevées. L'incidence de l'existence de ces deux races sur les programmes de sélection pour la résistance est discutée.

épidémiologie - inoculum primaire - résistance - pathotypes - sélection variétale

\begin{abstract}
Summary - Identification of 2 races of Powdery mildew (Erysiphe polygoni D.C.) on bean (Phaseolus vulgaris) in Guadeloupe. Greenhouse and field reactions of different varieties, naturally or artificially mildewed, suggested an heterogeneity of the pathogen population (Erysiphe polygoni D.C). Two races differing in virulence and agressiveness prevail during the cultural season (December to June on 1987-1988.). Both survive on wild legumes. Race 1 appears first on bean and requires cooler temperatures than race 2 for development. Race 2 comes later and has a wider spectrum. Expected consequences on breeding for resistance are discussed.
\end{abstract}

epidemiology - primary inoculum - resistance - pathotypes - selection

\section{Introduction}

L'effondrement de la résistance verticale des variétés est un phénomène que l'on doit redouter particulièrement chez les parasites disséminés par voie aérienne sur de longues distances et dont les épidémies évoluent selon un intérêt composé (Van Der Plank, 1963). La connaissance des races parasitaires et de leur dynamique est importante pour établir une bonne stratégie de sélection.

Contrastant par exemple, avec l'oïdium de l'orge (Erysiphe graminis f. sp. hordei), pour lequel de nombreux travaux ont permis de dénombrer plus d'une centaine de races physiologiques identifiables par une gamme de 10 variétés différentielles, l'oïdium du haricot, maladie spécifique du continent américain, a été peu étudié. Concernant les races, les travaux de Dundas (1941, 1942) font allusion à 3 , puis même 14 races dans un résumé, mais n'ont jamais été publiés de façon complète. L'existence de races différentes, suivant la localisation géographique, peut être perçue aux Antilles, où la variété "Contender" réputée résistante à l'oïdium dans les catalogues américains se révèle sensible. L'hypothèse d'une hétérogénéité locale de la population d'oïdium en Guadeloupe est perceptible pendant nos essais de serre ou de champ.

a) En serre, sur les plantules de la variété "Borlotto» servant aux inoculations, par la manifestation de deux dynamiques de l'oïdium, surtout pendant la phase d'incubation et d'apparition des premiers symptômes, réactions décrites par 
Messiaen (1989). L'une, «rapide», est observable en début de saison sèche, l'autre, «lente», est observable en fin de saison sèche et début de saison humide. Ce phénomène peut être interprété soit comme une réaction différente du parasite en fonction des conditions climatiques caractéristiques des 2 saisons du climat antillais, soit comme une modification de la population de l'oïdium.

b) Lors de la notation sous serre de certaines variétés comme CNR44, qui paraît résistante en début de saison (octobre, novembre) pendant la manifestation "rapide» de l'oïdium, puis sensible pendant la période de manifestation «lente».

c) Lors d'essais effectués au champ, la même variété CNR44 paraît moins attaquée en début de végétation, plus attaquée en fin de végétation; phénomène pouvant s'interpréter soit comme une variation de sensibilité de la plante suivant son stade physiologique, soit comme l'intervention progressive d'une autre race capable d'attaquer cette variété.

La connaissance de la structure des populations du parasite est indispensable à l'établissement de programmes de sélection pour la résistance; l'objet du travail présenté est d'y contribuer.

\section{Matériel et Méthodes}

\section{Matériel végétal}

Divers cultivars sélectionnés et déjà testés par Messiaen (1989) ont été utilisés. Les essais en serre ou en pièce climatique sont effectués en pots à section carrée de $10 \mathrm{~cm}$ de côté remplis de terre stérilisée. Les essais au champ sont réalisés selon des semis en lignes espacées de $40 \mathrm{~cm}$ effectués de décembre à février (pour assurer une récolte en grains avant la saison des pluies), les grains étant espacés de $5 \mathrm{~cm}$ sur la ligne. Les contaminations sont réalisées soit en intercalant des lignes de la variété italienne "Borlotto nano vigevano" (repérée par Messiaen pour sa grande sensibilité à l'oïdium), soit en mettant une bordure de cette même variété à l'est du champ (aux Antilles, le vent souffle presque en permanence de l'est-sudest), ce qui crée ainsi un gradient de maladie.

\section{Sources d'inoculum}

Une première source d'inoculum primaire est constituée au mois d'octobre à partir des feuilles d'une légumineuse sauvage : Pueraria phaseoloides (Roxb.) Benth, récoltées dans la nature et portant de l'oïdium surtout dans des endroits ombragés. Des feuilles malades sont secouées au-dessus de plantules de la variété "Borlotto vigevano". Cette variété semée hebdomadairement en serre permet de disposer d'un réservoir d'inoculum permanent pour les essais de serre, de pièce climatique et de champ. Les contaminations d'une série à l'autre se font naturellement sous l'effet du vent (les serres sont largement ouvertes sur les côtés) ou sont favorisées en soufflant quotidienne- ment sur des plantes très infectées au voisinage de la série suivante. Une autre source d'inoculum est constituée vers le mois de février en semant la variété CNR44 dans une serre éloignée de plus de $500 \mathrm{~m}$ à vol d'oiseau de la précédente; l'oïdium s'est progressivement installé sur les plantules de cette serre, constituant un autre réservoir d'inoculum primaire pour les contaminations.

\section{Protocole de mise en évidence des races}

Nous avons employé une gamme d'hôtes différentiels de 4 variétés : Borlotto vigevano +3 variétés sélectionnées par Messiaen et al. (1989) : CNR1, CNR44 et Salagnac 90 . Ces variétés sont cultivées en pot en pièce climatique à température quasiment constante de $22-23^{\circ} \mathrm{C}$, une photopériode de $12 \mathrm{~h}$ avec un éclairement de $10^{4}$ lux.

La veille de l'inoculation, les feuilles des plantes contaminatrices sont vigoureusement secouées afin de les débarasser de vieilles conidies non viables. Les jeunes conidies formées pendant la nuit servent à l'inoculation proprement dite par secouage des feuilles malades au-dessus des plantes à contaminer. La contamination est faite sur les deux tiers de l'effectif des plantes, le tiers restant servant de témoin. Les plantes contaminées sont mises immédiatement dans 2 pièces climatiques : pièce $n^{\circ} 1$ : température minimale de $23^{\circ} \mathrm{C}$, maximale $26^{\circ} \mathrm{C}$; pièce $\mathrm{n}^{\circ} 2$ : température minimale de $28^{\circ} \mathrm{C}$, maximale $35^{\circ} \mathrm{C}$.

Les observations sont ensuite faites matin et soir. On repère en particulier l'apparition de mycélium à la surface des feuilles et l'accomplissement de la période de latence (présence de conidiophores porteurs de conidies disséminables).

\section{Etude de la dynamique épidémique des races en fonction de la température}

L'effet de la température a été testé, d'une part, sur la germination des conidies, et, d'autre part, sur la durée de la période de latence et l'intensité de la sporulation.

\section{Germination des conidies}

Des plantes infectées préparées comme pour la contamination sont secouées au-dessus de lames porteobjet de microscope. Les lames sont déposées sur un support en verre dans une boîte de Petri contenant une solution aqueuse saturée en chlorure de sodium, assurant une humidité relative voisine de $75 \%$. Les boîtes sont ensuite placées dans 2 pièces réfrigérées par climatiseur, l'une ayant un minimum de $23^{\circ} \mathrm{C}$, un maximum de $26^{\circ} \mathrm{C}$ (conditions "fraîches"), l'autre un minimum de $28^{\circ} \mathrm{C}$, un maximum de $35^{\circ} \mathrm{C}$ (conditions "chaudes"). Le même éclairement ( $10^{4}$ lux) au niveau des lames est assuré dans les 2 pièces et la photopériode est de $12 \mathrm{~h}$. Le pourcentage de conidies germées est mesuré au bout de 24 h sur 5 lames, par conditions de températures, sur un effectif de 200 spores par lame. Toute conidie dont le tube germinatif dépasse la largeur de la spore est considérée comme germée. Une analyse de variance est effectuée sur les pourcentages après transformation de Bliss.

\section{Période de latence}

Les pustules sont examinées dès leur apparition, la présence de conidies décelée à l'aide d'une loupe compte-fil détermine la fin de la période de latence. 
Intensité de la sporulation

Ce paramètre n'est pas mesuré mais simplement estimé selon 3 classes : classe 1 : sporulation très faible à faible; classe 2 : sporulation moyenne; classe 3 : sporulation abondante.

L'utilisation de la technique employée par Pauvert (1976 et 1986) sur quelques échantillons de pustules ainsi que les indications de Pady et al. (1969) concernant la sporulation d'E. polygoni permettent d'établir approximativement que, dans la classe 1 , l'interisité de sporulation est inférieure à 10 conidies. $\mathrm{mm}^{-2}$.jour ${ }^{-1}$, comprise entre 10 et 100 conidies.mm-2.jour-1 dans la classe 2 , supérieure à 100 conidies. $\mathrm{mm}^{-2}$.jour-1 dans la classe 3.

\section{Résultats}

\section{Comportement de la variété Borlotto en serre}

La progression de l'oïdium sur les plantules est souvent irrégulière, surtout en octobre à partir de l'inoculum provenant de Pueraria phaseoloides. Cependant, à partir du 15 novembre, on assiste à un développement très rapide de l'ö̈dium. Environ une semaine après germination des graines, on observe sur les 2 premières feuilles des taches vert sombre donnant un aspect de mosaïque. Ces taches correspondent aux futures pustules contagieuses qui confluent par la suite et occupent toute la feuille. La maladie progresse ensuite sur les feuilles suivantes, qui se recroquevillent et n'atteignent pas leur taille normale. Des pustules se forment également sur les tiges. Par la suite, en particulier au début avril, l'aspect mosaïque devient moins visible puis absent et la progression de l'oïdium est plus lente. La densité de conidies dans les pustules est aussi plus faible qu'en décembre (classe 2 au lieu de 3).

\section{Comportement de la variété CNR44 en serre}

Sur cette variété, en février et en serre, l'oïdium s'installe progressivement, sans inoculation, par contamination extérieure. L'aspect mosaïque ne se manifeste pas, les pustules apparaissent progressivement et deviennent très poudreuses sous l'effet d'une sporulation abondante. L'examen de ces pustules au microscope confirme l'identité d'E. polygoni.

\section{Comportement d'essais variétaux au champ}

Les observations effectuées par Messiaen ainsi que les essais d'épidémies initiées par la variété Borlotto effectués au champ en février-mars 1987 et 1988 ont rapidement mis en évidence que la variété CNR44 paraissait, globalement, moins attaquée lorsqu'elle était semée en début de saison, et plus attaquée en fin de végétation qu'en début. Ce comportement pouvait s'expliquer soit par une variation de résistance de la plante au cours de sa période végétative, soit par l'intervention progressive sur cette variété d'une autre population-parasite virulente, qu'il convenait de mettre en évidence.

\section{Inoculations croisées}

Le Tableau I montre que, pour les expériences d'inoculation effectuées, l'inoculum provenant de Borlotto diffère de celui provenant de CNR44. L'inoculum provenant de Borlotto s'est révélé virulent sur son hôte d'origine et sur $\$ 90$, mais non virulent sur CNR1 et CNR44. L'inoculum provenant de CNR44 s'est révélé virulent sur les 4 variétés mais moins agressif sur Borlotto et $\mathrm{S} 90$; cette moindre agressivité se traduit par un allongement de la période de latence, une extension des symptômes plus lente et une sporulation moins forte. II est donc possible de parler d'une race 1 et d'une race 2 se différenciant à la fois par leur virulence et par leur agressivité.

\section{Influence de la température sur quelques para- mètres du cycle épidémique}

\section{Germination des conidies des 2 races}

La Figure 1 montre que les conidies de la race 2 supportent mieux les températures élevées que celles de la race 1. L'effet des températures plus chaudes que l'on rencontre pendant la saison humide (nette augmentation en particulier des températures nocturnes) peut expliquer que la compétition entre les 2 races sur les légumineuses sauvages comme Pueraria phaseoloides tourne à l'avantage de la race 2, dominante à partir d'avril, mai, juin. En revanche, lorsque les pluies diminuent et que les températures sont plus fraîches, les premières attaques s'effectuent avec la race 1.

Durée de la période de latence et intensité de la sporulation

Le Tableau II montre que l'allongement de la période de latence, quand on passe d'un régime

Tableau I. Pouvoir pathogène de l'inoculum provenant de Borlotto et CNR44 sur 4 variétés : résultats exprimés en heures de période de latence (précision $\pm 6 \mathrm{~h}$ ) à $23^{\circ} \mathrm{C}^{* * *}=$ aucun symptôme 8 jours après inoculation.

Origine de l'inoculum

\begin{tabular}{lcc} 
Variétés & Borlotto & CNR44 \\
\hline Borlotto & 72 & 96 \\
CNR44 & $\star \star \star$ & 72 \\
CNR1 & $\star \star \star$ & 72 \\
S90 & 72 & 96 \\
\hline
\end{tabular}




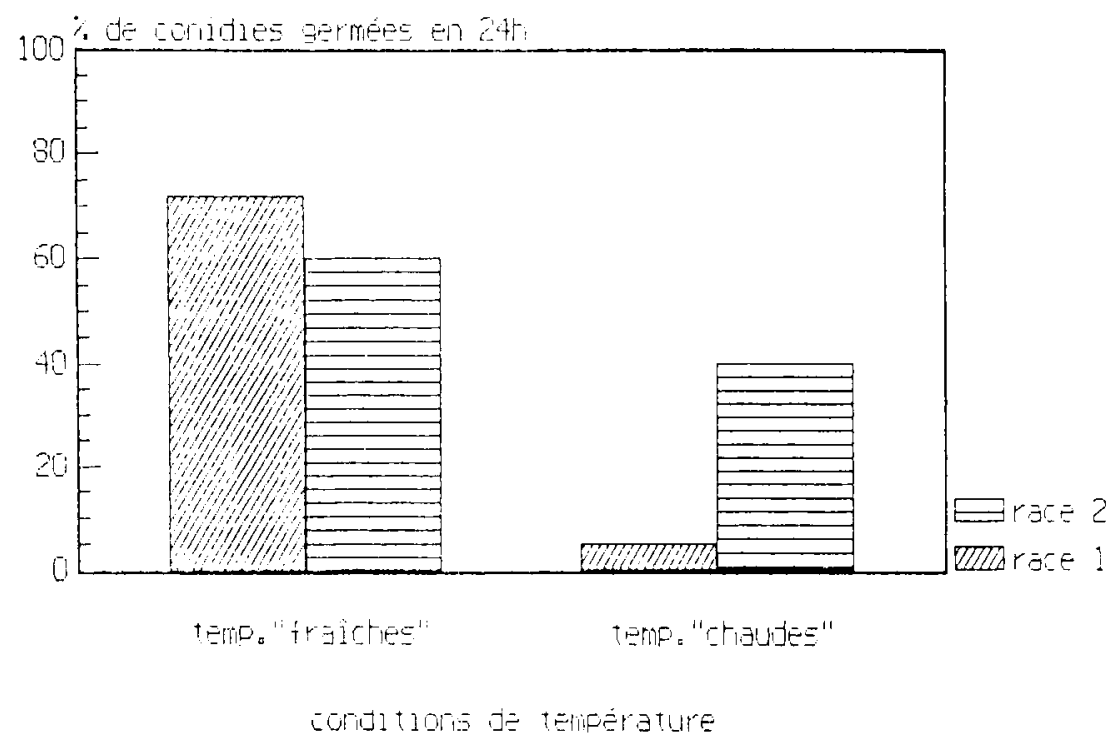

Fig. 1. Pourcentage de germination des conidies des 2 races en 24 h à 2 régimes de températures : températures «fraiches»: $\min .23^{\circ} \mathrm{C}-\max .26^{\circ} \mathrm{C}$; températures «chaudes": min. $28^{\circ} \mathrm{C}-\max .35^{\circ} \mathrm{C}$.

Tableau II. Durée de la période de latence (jours) et intensité de sporulation ( $2^{\mathrm{e}}$ chiffre : classe de sporulation) sur la variété Borlotto à 2 conditions de température.

\begin{tabular}{lcc} 
Tre en ${ }^{\circ} \mathrm{C}$ & Min : 23 Max :26 & Min : 28 Max : 35 \\
\hline Race 1 & $3(3)$ & $10(1)$ \\
Race 2 & $4(3)$ & $7(2)$ \\
\hline
\end{tabular}

de température "basses" à un régime de températures "plus élevées", est plus important dans le cas de la race 1 que dans celui de la race 2 . De plus, la sporulation de la race 1 est également plus faible. L'abaissement du taux de germination des conidies, l'augmentation de la durée de la période de latence ainsi que la diminution de l'intensité de la sporulation rendent la race 1 moins compétitive que la race 2 pendant la saison chaude.

\section{Discussion}

Des inoculations effectuées en pièce climatique vers le mois de juin à partir de légumineuses sauvages, en particulier Pueraria phaseoloides, ou de feuilles de haricots cultivés dans les environs mettent en évidence la race 2. Ces faits amènent les réflexions suivantes.

\section{En ce qui concerne la population parasite}

Erysiphe polygoni est présent sur de nombreuses légumineuses sauvages et cultivées. Le réservoir d'inoculum constitué en octobre pour les essais de serre et de champ à partir des légumineuses sauvages, Pueraria phaseoloides, Centrosema pubescens (Benth.), contient au départ la race 1 (ceci a été vérifié 2 années de suite). Cette race est virulente sur certaines variétés, alors que d'autres présentent une résistance absolue (CNR44 ou CNR1). La résistance de ces variétés est conférée par un gène dominant (Messiaen et al., 1989).

Au cours de la saison sèche, les attaques de plus en plus importantes sur les variétés telles que CNR44, que l'on observe tant en serre qu'au champ, que ce soit pour des épidémies naturelles ou initiées par la variété sensible «Borlotto", sont liées à l'installation progressive de la race 2 , dont la virulence semble très étendue, mais l'agressivité variable. Cette installation se fait au détriment de la race 1, tant sur haricot que sur les légumineuses sauvages que nous avons testées, en liaison avec l'augmentation des températures moyennes et surtout minimales (en Guadeloupe, l'augmentation des températures minimales est plus importante que celle des températures maximales quand on passe de la saison sèche à la saison humide). Les fortes pluies entraînent globalement une sévère diminution de l'oïdium, qui végète sur les légumineuses sauvages bien abritées, tant que la pluviométrie reste élevée.

\section{En ce qui concerne la population hôte}

La connaissance de la composition de la population-parasite et de son évolution est primordiale pour le sélectionneur. Les variétés pourvues de résistance absolues à la race 1 sont surtout utili- 
sables en début de saison sèche quand la race 2 n'est pas encore instaliée. Par la suite, les attaques de la race 2 affectent la majorité des variétés à des degrés divers. Dans ces conditions, l'utilisation des variétés présentant un bon niveau de résistance horizontale est primordiale. Ainsi, la variété $\$ 90$, attaquée par les 2 races, est sensible au stade plantule, mais présente une résistance en plein champ au stade adulte qui se manifeste par un net ralentissement de l'épidémie. Comme pour d'autres oïdiums, en particulier Erysiphe graminis D.C. f. sp. hordei, le "slow mildewing" est certainement le comportement à rechercher face à un parasite dont on connaît bien la grande variabilité génétique et la capacité à produire de nouvelles races.

D'autres études sont nécessaires pour préciser les modalités d'installation des races, l'existence éventuelle d'autres races, leur survie sur les légumineuses sauvages ou cultivées ainsi que leurs dynamiques respectives.

\section{Références}

Dundas B. (1941) Further studies on the inheritance of resistance to powdery mildew in beans. Hilgardia 13, 551-565

Dundas B. (1942) Breeding beans for resistance to powdery mildew and rust. Phytopathology 32, 828 (Abstract)

Messiaen C.M., Pauvert P., Jacqua G. \& Laraque A. (1989) L'oïdium américain du Haricot (Erysiphe polygoni D.C.) dans la zone antillaise : recherche de géniteurs de résistance. Agronomie 9, 3

Pady S.M., Kramer C.L. \& Clary (1969) Sporulation in some species of Erysiphe. Phytopathology 59, 844848

Pauvert P. (1976) Variations quantitatives de la sporulation d'Erysiphe graminis f. sp. hordei. Ann. Phytopathol. 8, 2, 131-140

Pauvert P. (1986) Les paramètres épidémiologiques de l'oïdium de l'orge (Erysiphe graminis $f$. sp. hordei). Thèse de doctorat, Université Pierre et Marie Curie (Paris VI)

Van Der Plank C.E. (1963) Plant Diseases; Epidemics and Control. Academic Press, New York and London 